\title{
Síndrome de Eagle tratado por medio de un abordaje transoral: reporte de caso
}

\author{
Eagle syndrome treated by a trans oral approach. Case report
}

Karen M. Reyes-Castillo ${ }^{*}$ y Jorge F. Moisés-Hernández ${ }^{2}$

'Servicio de Cirugía Oral y Maxilofacial, División de Estudios de Posgrado e Investigación, Facultad de Odontología, Universidad Nacional Autónoma de México; ${ }^{2}$ Servicio de Otorrinolaringología, Hospital General de México Dr. Eduardo Liceaga. Ciudad de México, México

\section{Resumen}

Antecedentes: El síndrome de Eagle es una condición poco común caracterizada por una alteración en la apófisis estilomastoides o una calcificación completa del ligamento estilohioideo. Objetivo: Reportar la relevancia de un diagnóstico oportuno y las opciones de tratamiento. Caso clínico: Mujer de 53 años con síntomas de cefalea occipital, dolor cervical y disfagia con 1 año de evolución. Por medio de imagenología y la clínica se establece el diagnóstico de síndrome de Eagle, que fue tratado con un abordaje transoral. Conclusiones: El síndrome de Eagle consta de signos clínicos inespecíficos, por lo que es importante comprender la evaluación diagnóstica y la variedad de opciones de tratamiento.

Palabras clave: Síndrome de Eagle. Apófisis estiloides. Abordaje transoral.

\section{Abstract}

Background: Eagle syndrome is an uncommon condition, characterized by alteration in the stylomastoid process and sometimes a complete calcification of the stylohyoid ligament. Objective: To report the relevance of a timely diagnosis and treatment options. Case report: 53-year-old female, present symptoms, characterized by occipital headache and neck pain and dysphagia with one-year evolution, through studies Imaging and clinical, the diagnosis of eagle syndrome was obtained, which is treated by surgical transoral approach. Conclusions: Eagle syndrome consists of nonspecific clinical signs, so it is important to understand the diagnostic evaluation, and the variety of treatment options.

Key words: Eagle Syndrome. Styloid process. Transoral approach.

\section{Introducción}

El síndrome de Eagle fue descrito por Watt Eagle en 1937 y es una condición clínica no común. Se estima que su frecuencia es de 4-8 por cada 10,000 personas y solo un $4-10.3 \%$ presentan sintomatología ${ }^{1,2}$.
La etiología del síndrome se ha reportado que puede ser multifactorial. Eagle propuso que una causa secundaria de alargamiento eran la cicatrización traumática y la hiperplasia relacionadas con una amigdalectomía previa'. En la actualidad se cree que el desarrollo óseo y la homeostasis contribuyen al

\section{Correspondencia:}

*Karen M. Reyes-Castillo

Circuito de los Institutos $\mathrm{s} / \mathrm{n}$, Ciudad Universitaria

Col. Copilco, Alcaldía Coyoacán

Fecha de recepción: 28-01-2021

C.P. 04510 , Ciudad de México, México

E-mail: kren_93_14@hotmail.com
Cir Cir. 2021;89(S2):41-44

Contents available at PubMed www.cirugiaycirujanos.com 
alargamiento de la apófisis estiloides, incluida la presencia de dos centros de osificación en la apófisis estiloides, la conversión embrionaria mesenquimal a matriz osteoide, cambios osteoartríticos y enfermedades relacionadas con el calcio y el fosfato ${ }^{3}$.

Este síndrome se caracteriza por un patrón de dolor en la región de la cabeza y el cuello que comúnmente incluye algunos de los siguientes síntomas: odinofagia, disfagia, acúfenos y dolor cervicofacial. Sin embargo, presenta una variedad de síntomas inespecíficos, ya que puede llegar a causar complicaciones potencialmente graves ${ }^{2,4-6}$.

El síndrome de Eagle puede confundirse con otras afecciones, como trastornos funcionales de la articulación temporomandibular o neuralgia glosofaríngea inespecífica y neuralgia del nervio occipital. En ocasiones, los pacientes con síndrome de Eagle reciben un diagnóstico erróneo durante mucho tiempo, lo que retrasa el tratamiento adecuado ${ }^{7,8}$. El tratamiento del síndrome de Eagle a veces requiere un manejo invasivo. Las opciones dentro de los tratamientos van desde el manejo conservador hasta el tratamiento quirúrgico, y hasta la fecha no existe consenso sobre el mejor abordaje para estos pacientes ${ }^{9-11}$.

El objetivo de este reporte es describir los signos clínicos y la evaluación diagnóstica de un caso, identificando las características del síndrome para brindar un manejo oportuno y adecuado.

\section{Caso clínico}

Mujer de 53 años que acude al servicio de cirugía maxilofacial del Hospital General de México, sin antecedentes sistémicos, alergia a medicamentos o intervenciones quirúrgicas en área cervical. Inicia su padecimiento aproximadamente hace 1 año con dolor localizado sobre la cara lateral del cuello del lado izquierdo, el cual se irradiaba hacia el oído y el ángulo mandibular homolateral, y ligero incremento del dolor con los movimientos del cuello. También refería sensación de cuerpo extraño en la garganta al deglutir. Acudió a diversos facultativos que indicaron múltiples tratamientos farmacológicos para su sintomatología, sin ninguna mejoría.

Se realizó una evaluación de los movimientos de extensión, flexión y rotación del cuello, y se palparon la pared anterior de la fosa amigdalina y la pared faríngea lateral del lado izquierdo, donde se produjo un episodio de dolor referido por la paciente.

En la tomografía computarizada se observó un alargamiento de la apófisis estiloides del lado izquierdo,

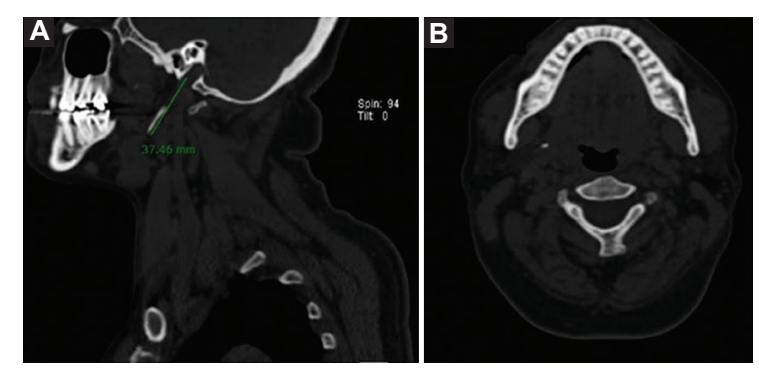

Figura 1. Tomografía computarizada en ventana ósea. Se aprecia el alargamiento anormal de las apófisis estiloides en el lado izquierdo. A: plano sagital correspondiente a una medida de $37 \mathrm{~mm}$. B: plano axial que muestra una imagen hiperdensa que se corresponde con las apófisis estiloides en el lado izquierdo.

de aproximadamente de $37 \mathrm{~mm}$, mientras que en el lado derecho no había ninguna elongación (Fig. 1). Por ello, de acuerdo con las características clínicas e imagenológicas, se estableció el diagnóstico de síndrome de Eagle.

El tratamiento de la paciente consistió en una intervención quirúrgica por abordaje intraoral modificado, bajo anestesia general balanceada, sin complicaciones posoperatorias (Figs. 2 y 3 ).

En el control clínico e imagenológico a los 12 meses, la paciente no presentó secuelas de parestesia ni daño a estructuras adyacentes, con supresión de la sintomatología dolorosa.

\section{Discusión}

El síndrome de Eagle es un diagnóstico poco frecuente, con una presentación poco variable, como fue el caso de la paciente; sin embargo, otros síntomas, como dolor de cabeza, pueden ser componentes de su presentación, aunque con menos frecuencia que el dolor cervical y la otalgia.

Es de importancia conocer la diversidad de los síntomas relacionados con el síndrome, ya que los pacientes diagnosticados erróneamente pueden someterse a tratamientos innecesarios e incluso a diversos procedimientos quirúrgicos, que incluyen extracciones dentales, reducciones de la tuberosidad, alveoplastias y artroscopias temporomandibulares, sin lograr ninguna mejoría clínica ${ }^{12}$.

El síndrome de Eagle se presenta con mayor frecuencia en mujeres, con una relación 3:13.

Se ha descrito que puede desarrollarse en pacientes que han sido sometidos a amigdalectomía, cuando el tejido cicatricial debajo de la fosa amigdalina comprime y estira los nervios craneales V, VII, IX y 


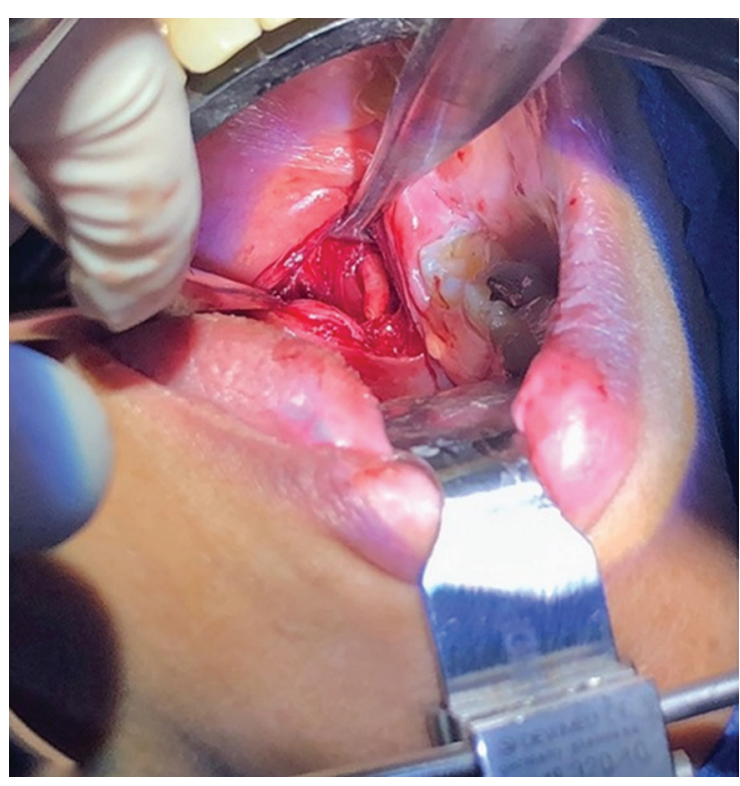

Figura 2. Vista intraoperatoria de la apófisis estiloides disecada.

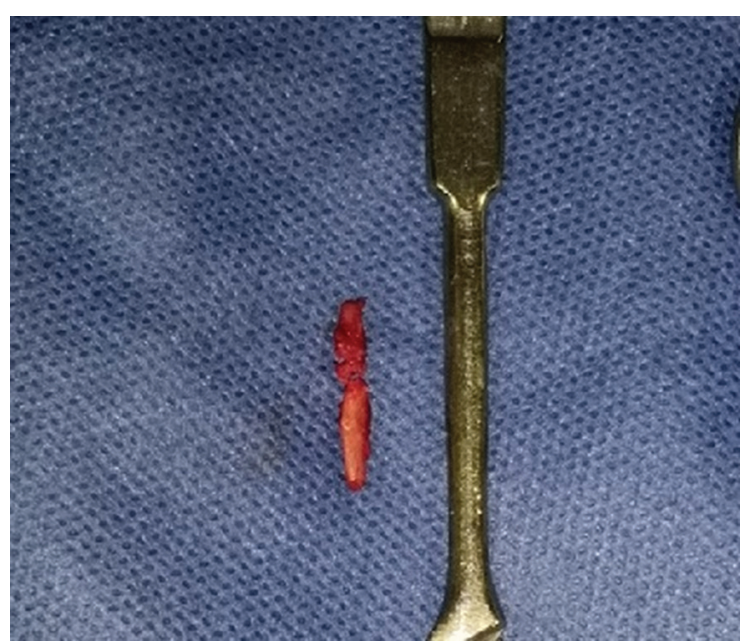

Figura 3. Vista intraoperatoria de la resección de la apófisis estiloides.

X; la irritación mecánica de un proceso alargado fijo o la presión sobre los nervios simpáticos y craneales puede provocar molestias. Esto puede ser exacerbado por la fibrosis posamigdalectomía ${ }^{14}$. En el caso reportado, la paciente no tenía antecedentes de amigdalectomía o trauma, lo que puede corrobora el hecho de que muchos casos de síndrome de Eagle son idiopáticos ${ }^{15}$.

Se han utilizado modalidades de imagen para el diagnóstico del síndrome, que incluyen proyección anteroposterior y posteroanterior del cráneo, cefalograma lateral, proyección oblicua lateral de la mandíbula, Towne y tomografía computarizada, la cual debe realizarse con un grosor de $5 \mathrm{~mm}$, preferiblemente con imágenes de reconstrucción coronal, sagital y volumétrica, ya que permite medir la longitud y la angulación de la apófisis estiloides y evaluar la relación con las estructuras anatómicas próximas ${ }^{16}$, y reduce el tiempo de diagnóstico.

El síndrome de Eagle se puede tratar de forma conservadora o quirúrgica, o ambas. La resección quirúrgica se ha aceptado en general como la modalidad de tratamiento principal del síndrome de Eagle. Por tanto, se han descrito enfoques transorales y extraorales para el tratamiento de la apófisis estiloides alargada.

El abordaje intraoral tradicional comienza con una amigdalectomía y se palpa la fosa amigdalina para identificar la punta de la apófisis estiloides. La disección se realiza a través de los músculos pterigoideo medial y constrictor superior. Se identifica la apófisis estiloides, se diseca el periostio y se eliminan los ligamentos de la apófisis. Por último, se utiliza una gubia para fracturar y retirar la punta de la estiloides lo más proximal posible ${ }^{9,11,17}$.

En un estudio de cohorte retrospectivo realizado por Hardin, et al. ${ }^{11}$ se informó que el abordaje transoral tendía a un tiempo operatorio significativamente más corto en comparación con el abordaje extraoral ${ }^{11}$.

Si bien algunos estudios favorecen el abordaje transoral por sus tiempos de operación reducidos, cicatrización mínima y riesgo reducido de lesión del nervio facial ${ }^{10,17}$, otros cirujanos prefieren el abordaje transcervical para una mejor visualización del campo quirúrgico (y quizás, por lo tanto, una resección más completa del proceso óseo) y un menor riesgo de infección; sin embargo, no es superior según algunos informes ${ }^{10,18}$.

También se han publicado abordajes intraorales por vía endoscópica y navegación quirúrgica, con la finalidad de disminuir la posibilidad de lesiones neurovasculares ${ }^{19,20}$.

\section{Conclusiones}

El síndrome de Eagle consta de signos clínicos inespecíficos, por lo que es importante comprender la evaluación diagnóstica y la variedad de opciones de tratamiento para la resolución de los síntomas.

\section{Agradecimientos}

Los autores agradecen al personal de los servicios de otorrinolaringología del Hospital General de México por todo el apoyo brindado. 


\section{Responsabilidades éticas}

Protección de personas y animales. Los autores declaran que para esta investigación no se han realizado experimentos en seres humanos ni en animales.

Confidencialidad de los datos. Los autores declaran que han seguido los protocolos de su centro de trabajo sobre la publicación de datos de pacientes.

Derecho a la privacidad y consentimiento informado. Los autores han obtenido el consentimiento informado de los pacientes y/o sujetos referidos en el artículo. Este documento obra en poder del autor de correspondencia.

\section{Financiamiento}

Los autores declaran que no hubo financiamiento.

\section{Conflicto de intereses}

Los autores no tienen conflictos de intereses que revelar.

\section{Bibliografía}

1. Eagle WW. Symptomatic elongated styloid process. Report of two cases of styloid process-carotid artery syndrome with operation. Arch Otolaryngol Head Neck Surg. 1949;49:490-503.

2. Kawasaki M, Hatashima S, Matsuda T. Non-surgical therapy for bilateral glossopharyngeal neuralgia caused by Eagle's syndrome, diagnosed by three-dimensional computed tomography: a case report. J Anesth 2012;26:918-21.
3. Fusco DJ, Asteraki S, Spetzl RF. Eagle's syndrome: embryology, anatomy, and clinical management. Acta Neurochirurgica. 2012;54:1119-26.

4. Costantinides F, Vidoni G, Bodin C, Di Lenarda R. Eagle's syndrome: signs and symptoms. Cranio. 2013;31:56-60.

5. Hebant B, Guegan-Massardier E, Macaigne V, Triquenot-Bagan A. Ischemic stroke due to internal carotid artery dissection associated with an elongated styloid process (Eagle syndrome). J Neurol Sci. 2017;372:466-7.

6. Todo T, Alexander M, Stokol C, Lyden P, Braunstein G, Gewertz B. Eagle syndrome revisited: cerebrovascular complications. Ann Vasc Surg. 2012;26:729.e1-5.

7. Thoenissen $P$, Bittermann $G$, Schmelzeisen R, Oshima T, Fretwurst $T$. Eagle's syndrome - a non-perceived differential diagnosis of temporomandibular disorder. Int J Surg Case Rep. 2015;15:123-6.

8. Pigache P, Fontaine C, Ferri J, Raoul G. Transcervical styloidectomy in Eagle's syndrome. Eur Ann Otorhinolaryngol Head Neck Dis. 2018;135:433-6.

9. Müderris T, Bercin S, Sevil E, Beton S, Kiris M. Surgical management of elongated styloid process: intraoral or transcervical? Eur Arch Oto-Rhino-Laryngol. 2014;271:1709-13.

10. Mortellaro C, Biancucci P, Picciolo G, Vercellino V. Eagle's syndrome: importance of a corrected diagnosis and adequate surgical treatment. J Craniofac Surg. 2002;13:755-8.

11. Hardin FM, Xiao R, Burkey BB. Surgical management of patients with Eagle syndrome. Am J Otolaryngol Head Neck Med Surg. 2018;39:481-4.

12. Bouguila J, Guiga B, Rejeb MB, Omezzine M, Mani R, Moatemri R, et al. Surgical treatment of Eagle's syndrome: transoral or cervical approach? Int J Oral Maxillofac Surg. 2015;44:e40-1.

13. Zeckler SR, Betancur AG, Yaniv G. The eagle is landing: Eagle syndrome — an important differential diagnosis. Br J Gen Pract. 2012;62:501-2.

14. Leong SCL, Karkos PD, Papouliakos SM, Apostolidou MT. Unusual complications of tonsillectomy: a systematic review. Am J Otolaryngol Head Neck Med Surg. 2007;28:419-22.

15. Bahgat $M$, Bahgat $Y$, Bahgat $A$. Eagle's syndrome, a rare cause of neck pain. BMJ Case Rep. 2012;2012:bcr2012006278.

16. Scavone G, Caltabiano DC, Raciti MV, Calcagno MC, Pennisi M, Musumeci AG, et al. Eagle's syndrome: a case report and CT pictorial review. Radiol Case Rep. 2019;14:141-5.

17. Torres AC, Guerrero JS, Silva HC. A modified transoral approach for carotid artery type eagle syndrome: technique and outcomes. Ann Otol Rhinol Laryngol. 2014;123:831-4

18. Mohanty S, Thirumaran NS, Gopinath M, Bambha G, Balakrishnan S. Significance of styloidectomy in Eagle's syndrome: an analysis. Indian J Otolaryngol Head Neck Surg. 2009;61:262-5.

19. Sukegawa $S$, Kanno $T$, Yoshimoto $A$, Matsumoto $K$, Sukegawa-Takahashi $\mathrm{Y}$, Masui $\mathrm{M}$, et al. Use of an intraoperative navigation system and piezoelectric surgery for styloidectomy in a patient with Eagle's syndrome: a case report. J Med Case Rep. 2017;11:1-6.

20. Spalthoff S, Zimmerer R, Dittmann O, Tavassol F, Dittmann J, Gellrich N-C, et al. Piezoelectric surgery and navigation: a safe approach for complex cases of Eagle syndrome. Int J Oral Maxillofac Surg. 2016;45:1261-7. 